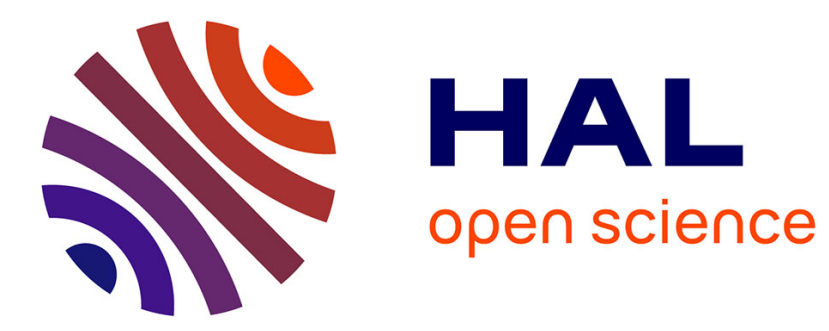

\title{
Energy partition in Sapphire and BGO scintillating bolometers
}

Y. Ortigoza, N. Coron, C. Cuesta, E. García, C. Ginestra, J. Gironnet, P. de Marcillac, M. Martínez, C. Pobes, J. Puimedón, et al.

\section{- To cite this version:}

Y. Ortigoza, N. Coron, C. Cuesta, E. García, C. Ginestra, et al.. Energy partition in Sapphire and BGO scintillating bolometers. Astroparticle Physics, 2011, 34 (8), pp.603. 10.1016/j.astropartphys.2010.12.013 . hal-00724417

\section{HAL Id: hal-00724417 https://hal.science/hal-00724417}

Submitted on 21 Aug 2012

HAL is a multi-disciplinary open access archive for the deposit and dissemination of scientific research documents, whether they are published or not. The documents may come from teaching and research institutions in France or abroad, or from public or private research centers.
L'archive ouverte pluridisciplinaire HAL, est destinée au dépôt et à la diffusion de documents scientifiques de niveau recherche, publiés ou non, émanant des établissements d'enseignement et de recherche français ou étrangers, des laboratoires publics ou privés. 


\section{Accepted Manuscript}

Energy partition in Sapphire and BGO scintillating bolometers

Y. Ortigoza, N. Coron, C. Cuesta, E. García, C. Ginestra, J. Gironnet, P. de Marcillac, M. Martínez, C. Pobes, J. Puimedón, T. Redon, M.L. Sarsa, L. Torres, J.A. Villar

PII: S0927-6505(11)00002-8

DOI: 10.1016/j.astropartphys.2010.12.013

Reference: ASTPHY 1561

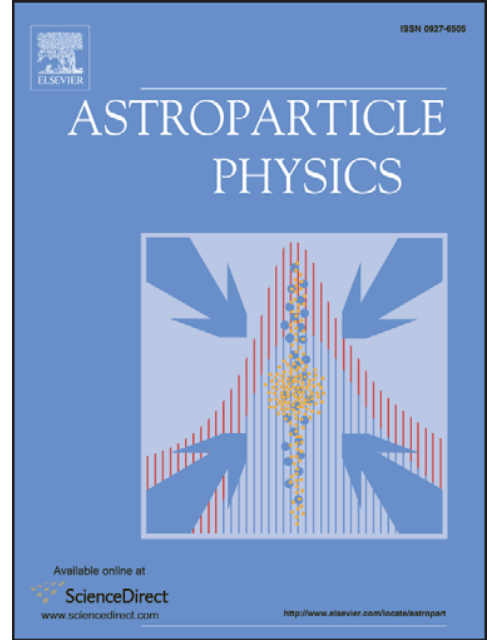

To appear in:

Astroparticle Physics

Received Date:

20 October 2010

Revised Date:

7 December 2010

Accepted Date:

30 December 2010

Please cite this article as: Y. Ortigoza, N. Coron, C. Cuesta, E. García, C. Ginestra, J. Gironnet, P. de Marcillac, M. Martínez, C. Pobes, J. Puimedón, T. Redon, M.L. Sarsa, L. Torres, J.A. Villar, Energy partition in Sapphire and BGO scintillating bolometers, Astroparticle Physics (2011), doi: 10.1016/j.astropartphys.2010.12.013

This is a PDF file of an unedited manuscript that has been accepted for publication. As a service to our customers we are providing this early version of the manuscript. The manuscript will undergo copyediting, typesetting, and review of the resulting proof before it is published in its final form. Please note that during the production process errors may be discovered which could affect the content, and all legal disclaimers that apply to the journal pertain. 


\title{
Energy partition in Sapphire and BGO scintillating bolometers
}

\author{
Y. Ortigoza ${ }^{\mathrm{a}, *}$, N. Coron ${ }^{\mathrm{b}}$, C. Cuesta ${ }^{\mathrm{a}}$, E. García ${ }^{\mathrm{a}}$, C. Ginestra ${ }^{\mathrm{a}}$, J. Gironnet $^{\mathrm{b}}$, P. de Marcillac ${ }^{\mathrm{b}}$, M. Martínez ${ }^{\mathrm{b}}$, \\ C. Pobes ${ }^{\mathrm{a}}$, J. Puimedón ${ }^{\mathrm{a}}$, T. Redon ${ }^{\mathrm{b}}$, M.L. Sarsa ${ }^{\mathrm{a}}$, L. Torres ${ }^{\mathrm{b}}$, J.A. Villar ${ }^{\mathrm{a}}$ \\ ${ }^{a}$ Grupo de Física Nuclear y Astropartículas. Universidad de Zaragoza. C/ Pedro Cerbuna 12, 50009 Zaragoza, Spain. \\ ${ }^{b}$ Institut d'Astrophysique Spatiale. Bâtiment 121, Université Paris-Sud 11 and CNRS (UMR 8617), 91405 Orsay Cedex, France.
}

\begin{abstract} luminescence properties of $\mathrm{BGO}$ at temperatures down to $77 \mathrm{~K}$.

Keywords:

Cryogenic detector, Scintillating bolometer, Energy partition, Light yield, Dark matter
\end{abstract}

Scintillating bolometers are particle detectors with a high particle discrimination power with many applications in nuclear and particle physics. This discrimination power is based on the different scintillation yield for different particles, and is strongly dependent on the target used. At the very low temperatures required for the operation of the bolometers, very few data about the scintillation yields are available. In this paper we present estimates of absolute light yields and energy partition among heat, light and trapping channels in Sapphire $\left(\mathrm{Al}_{2} \mathrm{O}_{3}\right)$ and $\mathrm{BGO}\left(\mathrm{Bi}_{4} \mathrm{Ge}_{3} \mathrm{O}_{12}\right)$ scintillating bolometers operated at $20 \mathrm{mK}$. The estimate relies on the observed negative correlation between the light and heat signals produced by $\gamma$-ray absorption in scintillating bolometers and on the study of the $\mathrm{x}$-ray stimulated

\section{Introduction}

Bolometers are cryogenic detectors widely used in the last decades (see for instance [1,2]) which measure as heat the energy deposited by particle interactions. They consist of an absorber where interacting particles deposit energy producing an increase of temperature $\Delta T$ and a thermal sensor (thermally connected to the absorber on one side and to a thermal bath via a heat leak on the other side) where $\Delta T$ is converted into an electrical signal. Massive bolometers can be built with dielectric and diamagnetic crystals cooled at temperatures of a few millikelvin because their heat capacity, which is only ascribed to vibrations of the crystal lattice and depends on the cube of the temperature, can be low enough to produce a measurable $\Delta T$.

The simultaneous measurement of heat and light signals was proposed in 1988 as a new tool for particle detection [3]: the very different scintillation yield of different particles $(\beta / \gamma$ particles, $\alpha$ particles and other recoiling nuclei) could be used to identify the type of particle, whereas the heat signal could allow to estimate the deposited energy with high resolution. The viability of this technique was shown in 1992 by the Milano group [4] using a Si photodiode glued on a $\mathrm{CaF}_{2}(\mathrm{Eu})$ bolometer to measure scintillation photons. In 1997 a French group [5] developed a new technique for the detection of scintillation photons using a second bolometer. This double bolometer technique is currently being used by the ROSEBUD Collaboration [6], the CRESST Dark Matter Search Experiment [7] and the Milano Group [8] and applied to rare event searches as double beta decay and dark matter.

ROSEBUD (Rare Objects SEarch with Bolometers UndergrounD) is a collaboration between the Universidad de Zaragoza and the Institut d'Astrophysique Spatiale (IAS), which develops and tests different materials as scintillating bolometer prototypes in order to apply them as detectors in nuclear and particle physics, with special emphasis on dark matter searches. Scintillating bolometers of $\mathrm{Al}_{2} \mathrm{O}_{3}, \mathrm{BGO}, \mathrm{CaWO}_{4}$ and $\mathrm{LiF}$ among others, have been developed and characterized by ROSEBUD (see for instance $[6,9]$ ). This work refers to a $50 \mathrm{~g} \mathrm{Al}_{2} \mathrm{O}_{3}$ and a $46 \mathrm{~g} \mathrm{BGO}$ scintillating

\footnotetext{
${ }^{*}$ Corresponding author. Tel.: +34 9767626 06; Fax: +34 976761247.

Email address: ortigoza@unizar.es (Y. Ortigoza)
} 


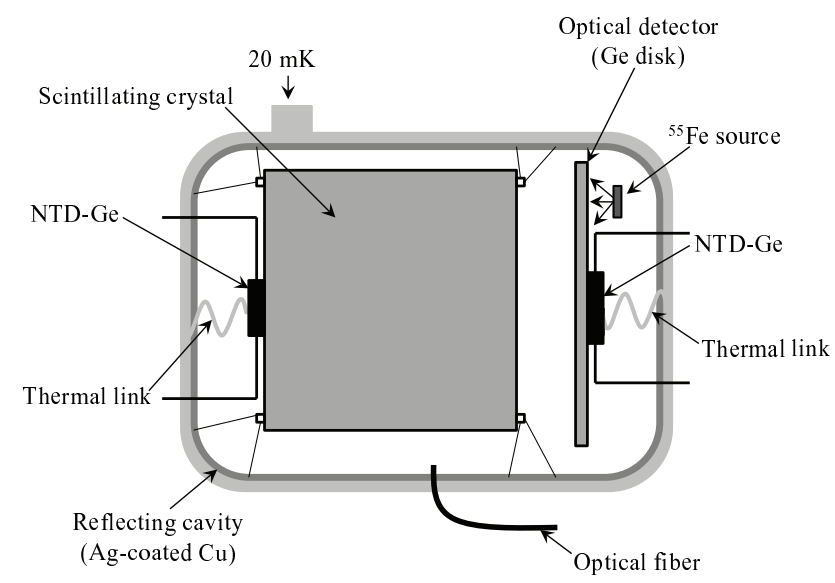

Figure 1: Schematic view of a double bolometer configuration. For the measurements presented in this work an inner ${ }^{55}$ Fe $\mathrm{x}-$ ray source was placed opposite the Ge bolometer to calibrate the energy absorbed on it.

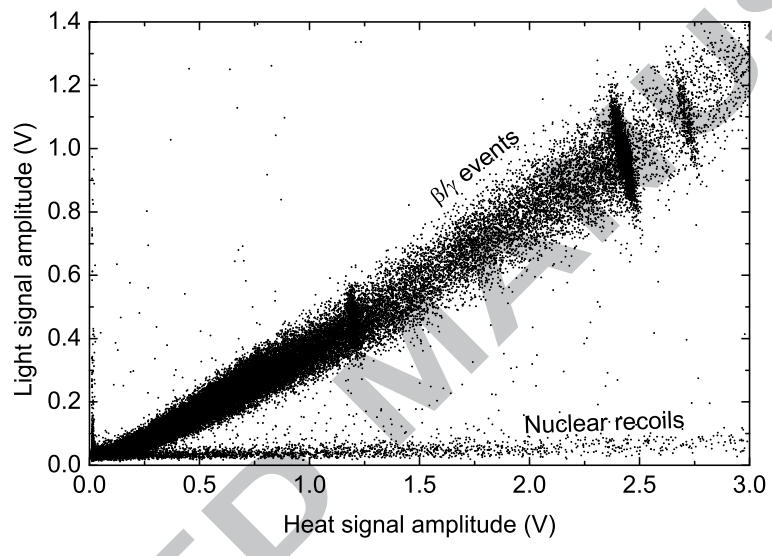

Figure 2: Light versus heat discrimination plot obtained with the $\mathrm{Al}_{2} \mathrm{O}_{3}$ scintillating bolometer [10]. The lines of 59.5 keV (from a ${ }^{241} \mathrm{Am}$ source), 122.1 and $136.5 \mathrm{keV}$ (from a ${ }^{57} \mathrm{Co}$ source) are clearly seen in the $\beta / \gamma$ band at values (in the heat channel) around $1.2,2.4$ and $2.7 \mathrm{~V}$ and show a negative correlation between the light and heat pulse amplitudes. A similar behaviour is observed in the BGO [11].

bolometers. As shown in figure 1, these scintillating bolometers consist of a scintillating crystal $\left(\mathrm{Al}_{2} \mathrm{O}_{3}\right.$ or $\left.\mathrm{BGO}\right)$ and a small Ge disk looking at the scintillating crystal, both mounted inside a copper cavity internally coated with $\mathrm{Ag}$ to obtain a good reflectivity. The scintillating crystal and the Ge disk have each one a neutron transmutation doped (NTD) Ge thermistor glued to it in order to measure thermal increases. The heat produced in the scintillating crystal is directly measured by its NTD-Ge sensor and the emitted light escaping from it that is eventually absorbed in the Ge crystal produces a thermal increase that is measured by the other NTD-Ge sensor. The double bolometers are thermally coupled to the mixing chamber of a dilution refrigerator operated at $20 \mathrm{mK}$. For technical details about the design, experimental set-up and operation of scintillating bolometers see $[10,11]$ and references therein.

Events in scintillating bolometers can be represented by points in a light versus heat pulse amplitude scatter plot. In these plots, monoenergetic events like those due to full absorption of $\gamma$-rays of a given energy appear as binormal distributions (see figure 2). Both channels, if independent, should not be correlated but we observed, first in a nominally pure Sapphire crystal [10] (with a measured Ti concentration of $6 \mathrm{ppm} \mathrm{[12])} \mathrm{and} \mathrm{then} \mathrm{in} \mathrm{a} \mathrm{nominally}$ pure BGO crystal [11], a negative correlation between the light and heat signals of these binormal distributions (see table 1) pointing at some energy transfer from one to the other channel. The same effect has also been noticed in a $\mathrm{CdWO}_{4}$ scintillating bolometer tested by members of the CUORICINO/CUORE Collaboration for Double Beta Decay experiments [13] and also in a dual phase liquid-gas Xe dark matter detector [14]. 
In reference [10] we proposed a quantitative interpretation of this correlation: for a given energy, the mean voltage outputs in the light and heat signals are directly related to the fraction of the deposited energy initially converted into light, $\alpha_{\ell}$ (we will refer to it as the absolute light yield), and the fraction of the deposited energy initially converted into heat, $\alpha_{h}$, respectively; the correlation coefficient originated by an energy transfer from one channel to the other is independent of $\alpha_{\ell}$ and $\alpha_{h}$; taking advantage of that, from these values one can estimate the ratio $\alpha_{\ell} / \alpha_{h}$. This method, developed for the $\mathrm{Al}_{2} \mathrm{O}_{3}$ scintillating bolometer was subsequently applied to the BGO scintillating bolometer [11]. Table 1 shows the values derived in both bolometers for the ratio $\alpha_{\ell} / \alpha_{h}$ at different $\gamma$-ray energies. One can observe that, in the range of energies analyzed, values obtained for $\alpha_{\ell} / \alpha_{h}$ in each scintillating bolometer are compatible with a constant value, independent of the energy, with weighted mean $0.144 \pm 0.010$ and $0.125 \pm 0.021$ for Sapphire and BGO, respectively.

Table 1: Correlation coefficient $(\rho)$ between heat and light pulse amplitudes on full absorption $\gamma$-ray lines and the derived ratio between the fraction of the deposited energy initially converted into light $\alpha_{\ell}$ and into heat $\alpha_{h}$ for scintillating bolometers of Sapphire and BGO operated at $20 \mathrm{mK}$. ${ }^{a}$ The line of $1633.3 \mathrm{keV}$ corresponds to a measurement made in different experimental conditions (lower gain). The first and second errors given for $\alpha_{\ell} / \alpha_{h}$ correspond to the statistic and systematic ones, respectively. Data taken from [10] for $\mathrm{Al}_{2} \mathrm{O}_{3}$ and [11] for BGO.

\begin{tabular}{cccc}
\hline & Energy $(\mathrm{keV})$ & $\rho$ & $\alpha_{\ell} / \alpha_{h}$ \\
\hline $\mathrm{Al}_{2} \mathrm{O}_{3}$ & 59.5 & $-0.61 \pm 0.02$ & $0.111 \pm 0.006 \pm 0.037$ \\
& 122.1 & $-0.890 \pm 0.005$ & $0.146 \pm 0.004 \pm 0.012$ \\
& 136.5 & $-0.87 \pm 0.01$ & $0.148 \pm 0.009 \pm 0.015$ \\
\hline $\mathrm{BGO}$ & 88.0 & $-0.24 \pm 0.02$ & $0.145 \pm 0.004 \pm 0.073$ \\
& 351.9 & $-0.39 \pm 0.05$ & $0.124 \pm 0.010 \pm 0.045$ \\
& 569.7 & $-0.29 \pm 0.03$ & $0.157 \pm 0.009 \pm 0.070$ \\
& 609.3 & $-0.27 \pm 0.07$ & $0.103 \pm 0.012 \pm 0.049$ \\
& 657.7 & $-0.54 \pm 0.06$ & $0.119 \pm 0.013 \pm 0.030$ \\
& $1633.3^{a}$ & $-0.21 \pm 0.08$ & $0.153 \pm 0.021 \pm 0.082$ \\
\hline
\end{tabular}

In these previous works, assuming that all the deposited energy is converted into heat and light (i.e., $\alpha_{\ell}+\alpha_{h}=1$ ), we have estimated absolute light yield for photons (0.127 \pm 0.010 in Sapphire [10] and $0.112 \pm 0.016$ in BGO [11]). This is an optimistic assumption because there are energy relaxation mechanisms (like, for instance, energy stored in traps living larger than a few milliseconds) that do not contribute to the heat or light signal, and, as explained in reference [10], estimates of the light yields should be properly rescaled (obtaining lower values). This paper reports the results of the study of the scintillation spectra of BGO down to $77 \mathrm{~K}$ which allows us to estimate the absolute light yield of BGO, without the assumption of absence of traps, and the light collection efficiency of its optical bolometer. From these values we have also estimated the energy partition (fraction of energy that goes into heat, light and traps) of both scintillating bolometers: Sapphire and BGO. The experimental set-up used in the study of the BGO scintillation spectra, the performed measurements and the results derived therefrom are discussed in the next sections.

\section{BGO scintillation spectra}

At IAS we have performed systematic measurements of the BGO scintillation spectra under $\mathrm{x}$-ray excitation from room temperature down to $77 \mathrm{~K}$. Figure 3 shows the experimental set-up used in these measurements. A small BGO crystal has been irradiated with photons from an $\mathrm{x}-$ ray tube $(40 \mathrm{kV}$ and $98 \mu \mathrm{A})$. The $\mathrm{BGO}$ crystal studied was a disk (2.06 $\mathrm{mm}$ thickness and $19.8 \mathrm{~mm}$ in diameter) mounted inside a copper holder (6 mm height and $21 \mathrm{~mm}$ in diameter) with the internal reflecting cavity coated with $\mathrm{Ag}$ (a similar mounting to that used in our scintillating bolometers). The holder was located inside a cryostat with a Be window for $\mathrm{x}$-ray irradiation. A thin $\mathrm{Al}$ foil on the top of the holder cavity was also facing to the Be window to allow photons from the $\mathrm{x}$-ray tube to reach the crystal. Scintillation photons were collected with an Avantes optical fiber UV 0706058 (2 m length, $1 \mathrm{~mm}$ in diameter, spectral band between 370 and $1070 \mathrm{~nm}$ and numerical aperture $N A=0.22$ ) coupled to the crystal cavity, and transmitted to an Avantes Fiber Spectrometer 2048 TEC where they were diffracted with a grating plane VA (VIS/NIR) of 300 lines $/ \mathrm{mm}$ and registered with a CCD detector. Figure 4 shows the BGO scintillation spectra measured at different temperatures while the BGO crystal was cooled from room temperature down to $77 \mathrm{~K}$. These spectra show that the number of scintillation photons emitted under $\mathrm{x}$-ray excitation increases as the temperature decreases and that at the lowest 
temperatures (below $150 \mathrm{~K}$ ) tends to a constant value in agreement with the behavior reported in [15] where the scintillation light response of BGO was measured down to a temperature of $6 \mathrm{~K}$. In reference [15], taking the light yield of BGO at room temperature to be $6900 \pm 140$ photons/MeV [16], they have estimated that the BGO light yield has a value of $23700 \pm 2600$ photons $/ \mathrm{MeV}^{1}$ at $6 \mathrm{~K}$. In figure 4 can be also observed a change in the shape of the spectra: the relative amplitude of the second peak at around $550 \mathrm{~nm}$ increases as temperature decreases and, as happens with the total light output, there is no noticeable change at the lowest temperatures (below $150 \mathrm{~K}$ ).
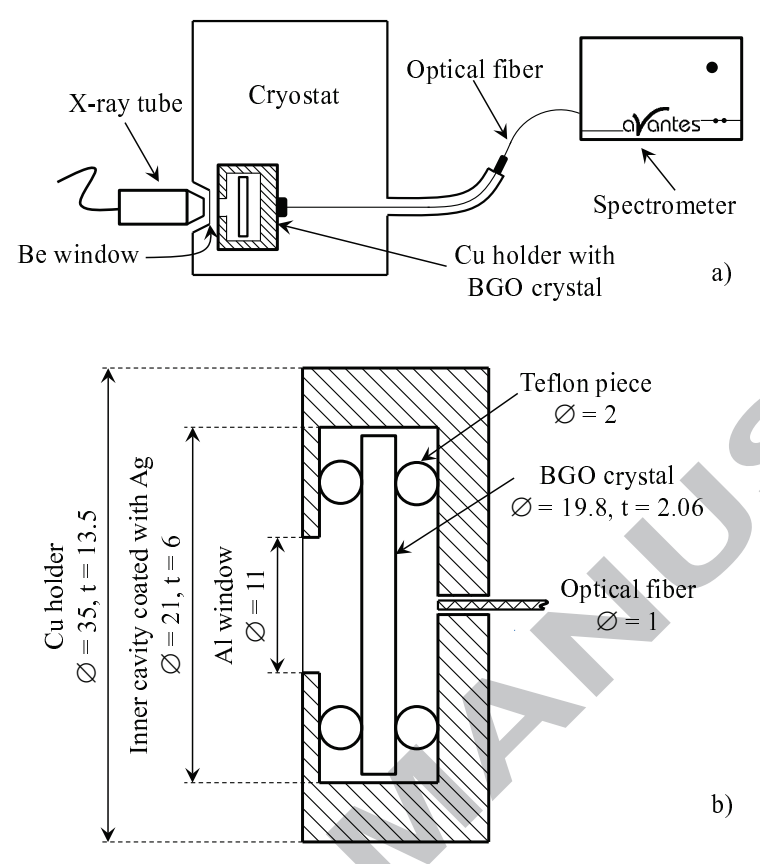

Figure 3: a) Experimental set-up of the cryostat and the crystal holder used for measuring the BGO emission spectra at different temperatures. b) Detail of the Cu holder with the BGO crystal. Here $\varnothing$ and $t$ stand for, respectively, the diameter and thickness dimensions (given in mm) of the different components.

From the measured spectrum $I_{m}(\lambda)$ at $77 \mathrm{~K}$, shown in figure 4, we can estimate the relative intensity of scintillation photons emitted $n(\lambda)$ at this temperature as

$$
n(\lambda)=\frac{I_{m}(\lambda)}{\eta_{c}(\lambda) \cdot \eta_{f}(\lambda) \cdot \eta_{g}(\lambda) \cdot S_{s}(\lambda)}
$$

where $\lambda$ is the wavelength, $\eta_{c}, \eta_{f}$ and $\eta_{g}$ are the transmission efficiencies of the crystal cavity, of the optical fiber and of the diffraction grating, respectively, and $S_{s}$ is the spectral relative sensitivity of the CCD detector (data on $S_{s}$ are only available from $400 \mathrm{~nm}$ to $700 \mathrm{~nm}$ but it practically covers the full BGO emission spectrum). Figure 5 shows the dependence with $\lambda$ of these four correction coefficients. Three of them $\left(\eta_{f}, \eta_{g}\right.$ and $\left.S_{s}\right)$ have been taken from the manufacturer's datasheets. The cavity transmission efficiency, $\eta_{c}$, is defined as the ratio between the light collected by the optical fiber $I_{c}(\lambda)$ and that emitted by the crystal $I_{e}(\lambda)$. This coefficient can be estimated in a simple model by

$$
\begin{gathered}
\eta_{c}(\lambda)=\frac{I_{c}(\lambda)}{I_{e}(\lambda)}=x+R(\lambda) \cdot(1-x) \cdot \varphi \cdot \sum_{n=0}^{\infty} R^{n}(\lambda) \cdot(1-\varphi)^{n} \\
\eta_{c}(\lambda)=x+\frac{R(\lambda) \cdot \varphi}{1-R(\lambda) \cdot(1-\varphi)} \cdot(1-x)
\end{gathered}
$$

\footnotetext{
${ }^{1}$ Notice that this value is consistent with that obtained at $77 \mathrm{~K}$ in [16] supporting the observed independence with temperature of the light yield below $100 \mathrm{~K}$.
} 


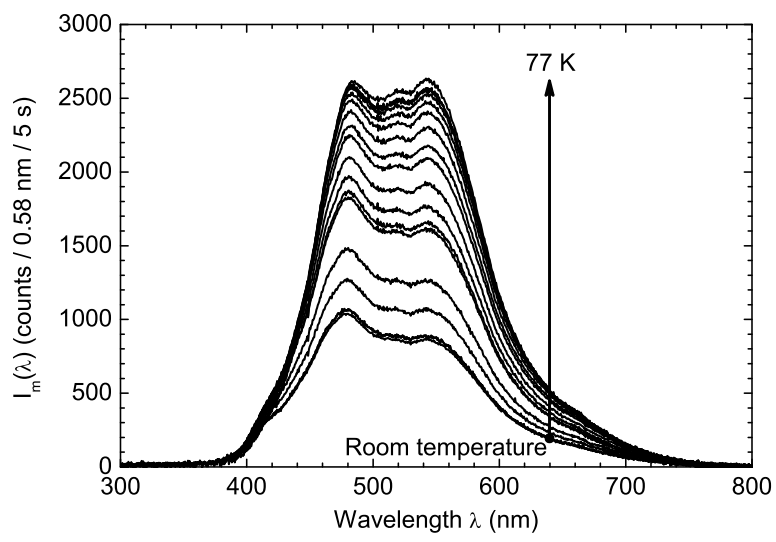

Figure 4: BGO differential scintillation spectra $I_{m}(\lambda)$ measured at different temperatures from room temperature down to $77 \mathrm{~K}$ with an $\mathrm{x}$-ray excitation source. All measurements were made with an integration time in the spectrometer of 5 s. Intermediate measurements were performed dynamically while the cryostat was cooling down, measuring the temperature of the surroundings of the crystal. These values are not given because they do not reflect the exact temperature of the crystal (it could be hotter). The last three-four intermediate spectra (with surroundings temperatures around $130-160 \mathrm{~K}$ ) and the $77 \mathrm{~K}$ spectrum show that there is no noticeable change in shape.

where the first term, $x$, represents the fraction of light directly collected by the optical fiber and the second one, the light collected after successive reflections in the cavity, being $R(\lambda)$ the reflectivity of the cavity coating [17] (also shown in figure 5) and $\varphi$ the probability that a photon reaches the fiber with a correct angle for transmission. With our geometry we have $x=\frac{S_{f}}{S_{c}} \frac{\Omega_{f}}{\Omega_{c}}$ and $\varphi=\frac{S_{f}}{S_{c}} \frac{\Omega_{f}}{2 \pi}$, where $S_{f}$ is the cross section of the optical fiber, $S_{c}$ is the cavity total area, $\Omega_{f} \approx \pi \cdot N A^{2}$ is the solid angle for transmission of photons by the fiber and $\Omega_{c}$ is the solid angle subtended by the crystal at the optical fiber position.

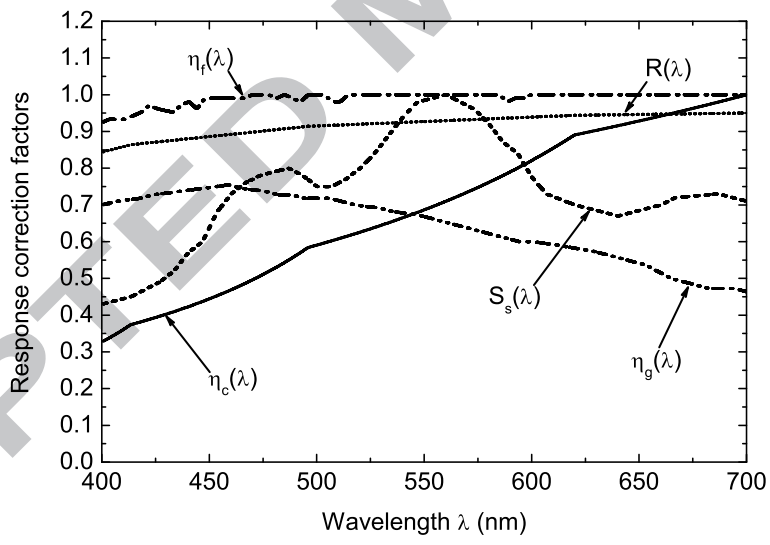

Figure 5: Transmission efficiencies of the crystal cavity $\left(\eta_{c}\right)$, of the optical fiber $\left(\eta_{f}\right)$, and of the diffraction grating $\left(\eta_{g}\right)$, and spectral relative sensitivity of the CCD detector of the spectrometer $\left(S_{s}\right)$ as a function of the wavelength. Also shown is the $\mathrm{Ag}$ reflectivity $R(\lambda)[17]$ used for the $\eta_{c}$ estimate.

Values derived for $\eta_{c}(\lambda)$ from equation 3 (normalized to one for $700 \mathrm{~nm}$ ) are shown in figure 5. Figure 6 shows the measured scintillation spectrum $I_{m}(\lambda)$ of BGO at $77 \mathrm{~K}$ (raw data) and the relative intensity of scintillation photons $n(\lambda)$ emitted at this temperature (corrected data) as obtained from equation 1 and normalized to $I_{m}(\lambda)$ area. The average energy of BGO scintillation photons at $77 \mathrm{~K}$ can be estimated from $n(\lambda)$ as

$$
\langle E\rangle=h c\left\langle\frac{1}{\lambda}\right\rangle=h c\left\{\frac{1}{N} \sum_{\lambda=\lambda_{i}}^{\lambda_{f}} \frac{1}{\lambda} \cdot n(\lambda)\right\}
$$


where $N=\sum_{\lambda=\lambda_{i}}^{\lambda_{f}} n(\lambda)$ and, in our case, $\lambda_{i}=400 \mathrm{~nm}$ and $\lambda_{f}=700 \mathrm{~nm}$. We estimate a value of $\langle E\rangle=2.436 \pm 0.017_{\text {stat }} \pm$ $0.068_{\text {syst }} \mathrm{eV}$, where the systematic error is introduced to take into account the small effects produced by the presence of the teflon pieces and the $\mathrm{Al}$ foil on top of the holder cavity, and also by the crystal autoabsorption. It has been estimated as the difference between the mean energy obtained with and without the correction factors inclusion $(2.436 \pm 0.017 \mathrm{eV}$ and $2.368 \pm 0.017 \mathrm{eV}$ ). Assuming that, as reported by [15, 18], below $100 \mathrm{~K}$ the scintillation response remains practically constant and, as suggested by our results shown in figure 4, the BGO scintillation spectrum practically does not change at low temperatures, we can take this value as the average energy of BGO scintillation photons below $100 \mathrm{~K}$ and, in particular, at $20 \mathrm{mK}$ (the operation point of our bolometers). In fact, our estimate of $\langle E\rangle$ is in good agreement with studies of BGO luminescence at temperatures of a few Kelvin: for instance, in [19] operating at $10 \mathrm{~K}$ they report a broad luminescence band at $2.46 \mathrm{eV}$, whereas in [20] they observe at $5 \mathrm{~K}$ an intense emission band at around $510 \mathrm{~nm}(2.43 \mathrm{eV})$. This comparison suggests that practically the same scintillation mechanism and, consequently, the same scintillation response are observed in the different BGO crystals analyzed in the literature (see for instance [21-23]).

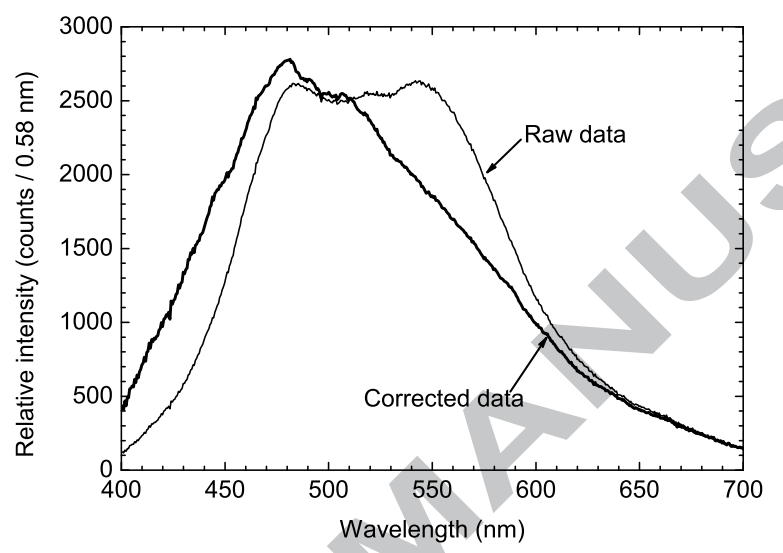

Figure 6: Raw data stands for the measured spectrum $I_{m}(\lambda)$ for BGO at $77 \mathrm{~K}$ and corrected data for the relative intensity of scintillation photons emitted at this temperature (obtained after corrections explained in the text and normalized to the same area of $I_{m}(\lambda)$ ).

\section{Light yield and energy partition in scintillating bolometers}

From the average energy of $\mathrm{BGO}$ scintillation photons $\langle E\rangle$ estimated in the previous section and the BGO light yield of $23700 \pm 2600$ photons/MeV at $6 \mathrm{~K}$ quoted by [15], we obtain that the absolute light yield of the BGO at $20 \mathrm{mK}$ is $\alpha_{\ell}=0.058 \pm 0.006_{\text {stat }} \pm 0.002_{\text {syst }}$.

Carrying out an absolute energy calibration of the light signal with an inner ${ }^{55} \mathrm{Fe} x$-ray source placed opposite to the optical Ge bolometer of the BGO scintillating bolometer, we can also estimate its light collection efficiency. We have observed that an $\mathrm{x}$-ray photon of $5.9 \mathrm{keV}$ from the ${ }^{55} \mathrm{Fe}$ source fully absorbed in the Ge bolometer produces the same light pulse amplitude that a photon of $841.3 \pm 8.3 \mathrm{keV}$ fully absorbed in the BGO scintillating crystal. Neglecting the fraction of the $\mathrm{x}$-ray energy lost in traps in the Ge target, we derive an equivalent light energy of $7.01 \pm 0.07 \mathrm{keV} / \mathrm{MeV}$ and a light collection efficiency of $0.121 \pm 0.013_{\text {stat }} \pm 0.004_{\text {syst }}$ for the double bolometer mounting. Notice that the Ge bolometer shows an excellent linearity as optical detector of the BGO in the range from 88 to $1633.3 \mathrm{keV}$ [24].

In the case of the Sapphire bolometer, since we do not have a measurement of the absolute light yield at low temperatures (of the order of a few Kelvin) for this Ti concentration (measurements at $9 \mathrm{~K}$ for others Ti concentration are given in [25]), we do conversely: from the light collection efficiency we estimate the absolute light yield. Since both scintillating bolometers have a very similar geometry, reflecting cavity ( $\mathrm{Ag}$-coated $\mathrm{Cu}$ ) and optical detector, we have assumed that light collection efficiency is the same. On the assumption that Ag reflectivity (ranging from 85 to $95 \%$, as shown in figure 5) is the dominant effect, influence of the different detector material and surface has been neglected. The absolute energy calibration of the optical Ge bolometer of the Sapphire scintillating bolometer with 
the ${ }^{55} \mathrm{Fe} \mathrm{x}$-ray source leads to an equivalent light energy detected of $13.5 \pm 0.3 \mathrm{keV} / \mathrm{MeV}$ and, consequently, to an

\section{Conclusions} absolute light yield $\alpha_{\ell}=0.112 \pm 0.012_{\text {stat }} \pm 0.004_{\text {syst }}$.

Finally, from the values of the absolute light yields and those derived for $\alpha_{\ell} / \alpha_{h}$ (see table 1), given that $\alpha_{\ell}+\alpha_{h}+$ $\alpha_{0}=1$ (where $\alpha_{0}$ is the fraction of deposited energy trapped), it is straightforward to estimate the deposited energy partition after photon interactions in $\mathrm{Al}_{2} \mathrm{O}_{3}$ and $\mathrm{BGO}$ scintillating bolometers (see table 2). Absolute light yield of Sapphire is twice that of BGO, whereas the trapping fraction is four times smaller. Notice that the scintillation yield of this Sapphire $(\approx 11 \%)$ is comparable with the values obtained with the most pre-eminent materials used as scintillation detectors at room temperature (for instance, thallium-activated sodium iodide reports light yield of about $\approx 12 \%$ [26]). This light response converts this sapphire into an excellent detector with particle discrimination capability down to energies of about $10 \mathrm{keV}$ [27]. The high $\alpha_{\ell}$ value of this Sapphire can be attributed, according to reference [28], to the presence of Ti in the crystal lattice.

Table 2: Energy partition in Sapphire and BGO scintillating bolometers for $\gamma$-ray interactions. Here are shown values obtained for the fraction of energy deposited that initially is converted into light $\left(\alpha_{\ell}\right)$, heat $\left(\alpha_{h}\right)$ or trapped $\left(\alpha_{0}\right)$. The first and second errors given correspond to the statistic and systematic ones, respectively.

\begin{tabular}{ccc}
\hline & $\mathrm{Al}_{2} \mathrm{O}_{3}$ & $\mathrm{BGO}$ \\
\hline$\alpha_{\ell}$ & $0.112 \pm 0.012 \pm 0.004$ & $0.058 \pm 0.006 \pm 0.002$ \\
$\alpha_{h}$ & $0.778 \pm 0.099 \pm 0.028$ & $0.464 \pm 0.092 \pm 0.016$ \\
$\alpha_{0}$ & $0.110 \pm 0.100 \pm 0.028$ & $0.478 \pm 0.092 \pm 0.016$ \\
\hline
\end{tabular}

We have measured the scintillation spectra of BGO at different temperatures from room temperature to $77 \mathrm{~K}$. From these spectra we have observed that the light output of the crystal increases and the spectral shape changes when cooling down. However, at the lowest temperatures both the light output and the spectral shape remain practically constant. Under this assumption, we have derived an average energy of $2.436 \pm 0.017_{\text {stat }} \pm 0.068_{\text {syst }} \mathrm{eV}$ for scintillation photons emitted by BGO at low temperatures. This value allows us to estimate the absolute light yield $\left(\alpha_{\ell}=0.058 \pm\right.$ $\left.0.006_{\text {stat }} \pm 0.002_{\text {syst }}\right)$ and the light collection efficiency $\left(0.121 \pm 0.013_{\text {stat }} \pm 0.004_{\text {syst }}\right)$ of the BGO scintillating bolometer and, indirectly, under the assumption of identical light collection efficiency, the absolute light yield of the Sapphire $\left(\alpha_{\ell}=0.112 \pm 0.012_{\text {stat }} \pm 0.004_{\text {syst }}\right)$. Combining these results with the ratio $\alpha_{\ell} / \alpha_{h}$, obtained from the negative correlation found between the light and heat responses in Sapphire and BGO scintillating bolometers, we have estimated the energy partition produced in both detectors for photon interactions: the fraction of deposited energy that initially goes into light, heat and traps.

\section{Acknowledgments}

This work has been supported by the Spanish Ministerio de Ciencia e Innovación (MICINN) (FPA2008-03228 and MULTIDARK Consolider CSD2009-00064), the French CNRS/INSU (MANOLIA and BOLERO projects) and the EU project ILIAS (RII3-CT-2004-506222). Y. Ortigoza was also supported by a UZ/BSCH/Fundación Carolina grant and by a DECRYPT (Detectores de Radiación y Partículas) investigation project contract. L. Torres is supported by a MICINN postdoctoral grant.

\section{References \\ References}

[1] K. Pretzl, Nucl. Instr. and Meth. A 454:1 (2000) 114.

[2] K. Pretzl, J. Phys.: Conf. Ser. 160 (2009) 012001.

[3] L. González-Mestres, D. Perret-Gallix, in Proceedings of "the 2nd European Workshop on Low Temperature Devices for the Detection of Low Energy Neutrinos and Dark Matte", 1988, p. 1.

[4] A. Alessandrello et al., Nucl. Phys. B (Proc. Suppl.) 28 (1992) 233. 
165 [5] C. Bobin, I. Berkes, J.P. Hadjout, N. Coron, J. Leblanc, P. de Marcillac, Nucl. Instr. and Meth. A 386 (1997) 453.

166 [6] N. Coron et al., in Proceedings of "Identification of Dark Matter 2008", PoS(idm2008)007.

167 [7] R.F. Lang, W. Seidel, New J. Phys. 11 (2009) 105017.

168 [8] L. Gironi, Nucl. Instr. and Meth. A 617 (2010) 478.

169 [9] S. Cebrián et al., Astropart. Phys. 21 (2004) 23.

170 [10] J. Amaré et al, Appl. Phys. Lett. 87 (2005) 264102.

171 [11] N. Coron et al., Opt. Mat. 31 (2009) 1393.

172 [12] M. Luca. PhD thesis, Université Claude Bernard-Lyon 1, 2007, http://hal.archives-ouvertes.fr/docs/00/18/23/26/PDF/thesisluca.pdf.

173 [13] C. Arnaboldi, J.W. Beeman, O. Cremonesi, L. Gironi, M. Pavan, G. Pessina, S. Pirro, E. Previtali, Astropart. Phys. 34 (2010) 143.

174 [14] E. Aprile, K.L. Giboni, P. Majewski, K. Ni, M. Yamashita, Phys. Rev. B 76 (2007) 014115.

175 [15] J. Gironnet, V.B. Mikhailik, H. Kraus, P. de Marcillac, N. Coron, Nucl. Instr. and Meth. A 594 (2008) 358.

176 [16] M. Moszyński, M. Balcerzyk, W. Czarnacki, M. Kapusta, W. Klamra, A. Syntfeld, M. Szawlowski, IEEE Trans. Nucl. Sci. NS51 (2004) $177 \quad 1074$.

178 [17] M. Bass, Handbook of Optics Vol. II, second edition, 1995.

179 [18] M. Itoh, T. Katagiri, J. Phys. Soc. Jpn. 79:7 (2010) 074717.

180 [19] K. Mori, H. Nishimura, M. Nakayama, Nonlinear Opt. 29:10-12 (2002) 609.

181 [20] M. Itoh, T. Katagiri, H. Mitani, M. Fujita, Y. Usuki, Phys. Stat. Sol. (b) 245:12 (2008) 2733

182 [21] M.J. Weber, R.R. Monchamp, J. Appl. Phys., 44:12 (1973) 5495.

183 [22] R. Moncorgé, B. Jacquier, G. Boulon, J. Lumin. 14 (1976) 337.

184 [23] V.Y. Ivanov, A.V. Kruzhalov, V.A. Pustovarov, V.L. Petrov, Nucl. Instr. and Meth. A 261 (1987) 150.

185 [24] Y. Ortigoza, PhD thesis, Universidad de Zaragoza, 2010.

186 [25] V.B. Mikhailik, H. Kraus, M. Balcerzyk, W. Czarnacki, M. Moszyński, M.S. Mykhaylyk, D. Wahl, Nucl. Instr. and Meth. A 546 (2005) 523.

187 [26] G.F. Knoll, Radiation Detection and Measurement, Third Edition, John Wiley \& Sons, Inc., 2000.

188 [27] J. Amaré et al., J. Phys.: Conf. Ser. 39 (2006) 133.

189 [28] M. Luca, N. Coron, C. Dujardin, H. Kraus, V.B. Mikhailik, M.-A. Verdier, P.C.F. Di Stefano. Nucl. Instr. and Meth. A 606 (2009) 545. 